\title{
H-infinity optimal control for micro-force sensing
}

\author{
Abdenbi Mohand Ousaid, Sinan Haliyo, Stéphane Régnier and Vincent Hayward
}

\begin{abstract}
This paper describes a micro-force sensing device where a H-infinity control scheme is used to actively balance the external force and provide the measurement. The force sensing principle is based on the active control of the electrostatic bipolar actuator (comb-drive) within the sensor. An external load force acts on the sensor and displaces the probe. The controller is set to balance the applied force and keep the probe at its null position. As a result, the applied force is obtained in real time from the balancing control signal. A meso-scale prototype has been built as proof-of-concept and an especially designed $\mathrm{H}$-infinity control allows for a measurement accuracy of $0.4 \mu \mathrm{N}$ over a large range $[-400+400] \mu \mathrm{N}$.

Index Terms-microrobotics, H-infinity control, force mea-
\end{abstract} surement, comb drive.

\section{INTRODUCTION}

The measurement of interaction forces is a key function in micro-scale probing, manipulation, and assembly systems [1]. The required range of displacement and forces vary greatly as a function of the intended application [2]. With small, dry objects, the van der Waals forces that are responsible for adhesion occur in $\mu \mathrm{N}$ range [3]. In the study of living systems, useful interactions can also occur at different length and force scales [4]. As an example, the study of contractile ventricular heart cells (cardiac myocytes), with a view to analyze the functional behavior of healthy or diseased cells, requires measurements to be made in the $\mu \mathrm{N}$ range [5].

Several types of micro and nano force sensors were developed to provide small force measurements. Many designs are based on the deformation of cantilevers [6], where the deformations can be detected using a variety of approaches, such as piezoresistance [7], capacitive sensing [8] and piezoelectricity [9]... Notably, in Atomic Force Microscopy (AFM), a cantilever is the tool of choice for force measurements [10], [11].

Despite their popularity, cantilevers have limitations that restrict the type of measurements that can be made. Cantilevers are hard to calibrate. In addition geometry-dependent probe/sample interactions cause the occurrence of a combination of bending and torsion deformation modes that can make data interpretation difficult. Other limitations can include the presence of several type of non-linearity, such as hysteresis and input saturation, that also limit the performance of cantilever-based devices. Perhaps the greatest limitation of the cantilevered beam is the unavoidable tradeoff that must be made between stiffness, sensitivity, and structural response characteristics. In practice, at a micro-

The authors are with Institut des systèmes Intelligents et de Robotique, Université Pierre et Marie Curie, CNRS UMR 7222, 4 Place Jussieu, 75252 Paris Cedex, France \{mohand ousaid, haliyo, regnier, hayward\}eisir.upmc.fr scale, a cantilever should be at the same time long to handle pull-in and and short to handle pull-off forces.

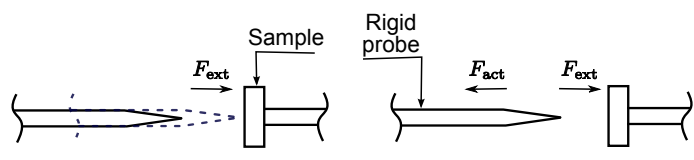

(a) Before balance

(b) After balance

Fig. 1. Force balance or compensation principle.

One approach to overcome these constraints is to employ feedback. Feedback can be used to cause a system to track a desired state, to become more robust to disturbances, or to stabilize an unstable system. But feedback has also the ability to provide a system with useful properties for sensing, larger bandwidth, better linearity, or a desired impedance presented to a measured object. Another great advantage of feedback is to make it possible to employ the same transducer as a sensor and an actuator. As shown in Fig 1, if a external force $F_{\text {ext }}$ attracts a rigid probe, the variation of the displacement of the probe is recorded and fed back to the actuator through a controller. An opposite force, $F_{\text {act }}$ is then generated to balance (compensate) the applied force and maintain the probe at equilibrium position. The magnitude of the external force can be derived from the applied voltage on the actuator.

Examples abound in the natural and the technological world, see for instance of accelerometers and pressure sensors [13], [14]. In those devices, an actuator is integrated to balance the externally applied forces seen as unknown input disturbances, keeping the moving parts in a state of static equilibrium. Following this approach, Sun and al [15] developed an active sensors capable of detecting microforces, in which interaction applied force is balanced using force generated by electrostatic micro actuator within the sensor. Shen and all [16] proposed a sensor design based on a cantilevered composite beam with embedded piezoelectric polyvinylidene fluoride layers (PVDF) for actuation and sensing, yet the beam stiffness trade-off was not eliminated in those designs.

In this paper, a sensor using electrostatic energy instead of elastic energy is presented and $\mathrm{H}$-infinity control approach enabled micro-force sensing is discussed. The developed sensor is a rigid probe constrained along one single dimension by a fiber suspension that provides nearly perfect kinematic guidance. Precise guidance makes it possible to measure the displacement of the moving probe carrier by laser. The suspended probe carrier included a linearised differential electrostatic actuator acting in the normal direction. By using $\mathrm{H}$-infinity control the probe is maintained at its equilibrium position, in order to balance the external force. Experimental 
tests are carried out to verify and validate the balance behavior of the sensor.

\section{FORCE SENSOR DESCRIPTION}

The sensor used in this work is an improved version of the device presented in [17], with one degree of freedom. It is designed around a moving probe kinematically constrained to a single translation motion, through a glass fiber suspension design, and carrying the moving part of a comb-drive armature.

\section{A. Fiber suspension}

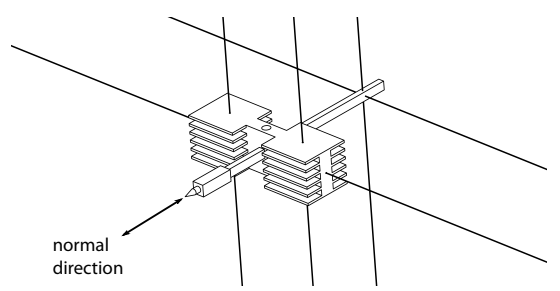

Fig. 2. Five-fiber suspension.

As depicted in Fig. 2, a suspension with fibres is used to constrain the motion of the probe to one degree of freedom (single axis motion). The 3 vertical fibers effectively constrain the motion of the carrier to planar motions. The 2 horizontal fibers add additional constrains to limit the motion to a single translation. The suspension compliance is tuned by adjusting the tension of the fibers and the tension in the front fibers equalize spurious torque. An optical solution, consisting of an external laser source, a mirror embedded on the carrier, and an external four quadrant photodiode is used to measure the displacement of the probe. The resulting system is shown in Fig. 3, including fiber fixations and the optical solution.

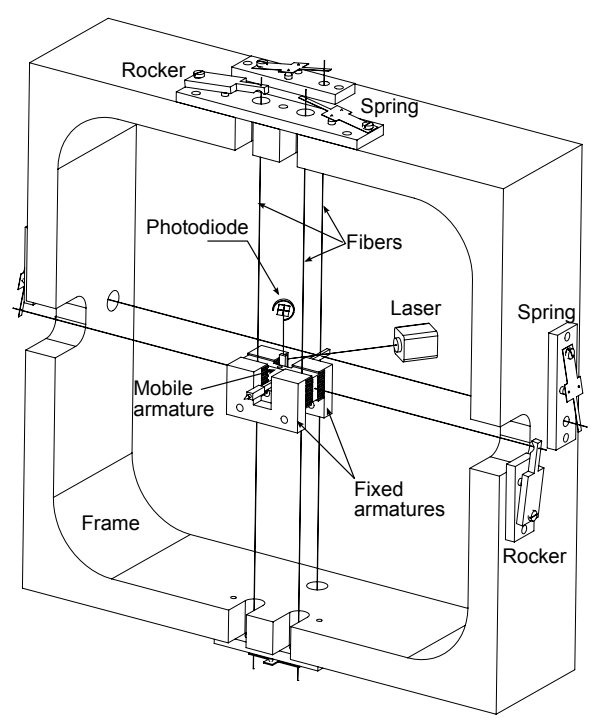

Fig. 3. Mechanical system.
(2)

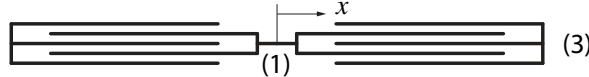

Fig. 4. Differential bipolar comb actuator.

\section{B. Differential bipolar comb actuator}

A bipolar differential electrostatic actuator (Comb-drive), is used in the system to balance the external force. Fig 4 depicts a schematic representation of such an actuator. A moving armature (1) is included in the carrier, and two fixed armatures (2) and (3) are mounted on the external frame. Given three voltages $v_{1}, v_{2}, v_{3}$ corresponding to the armatures (1), (2), and (3), the force developed by the actuator, given in [17] is :

$$
a_{n}=2 c_{n} v_{1} v_{n}
$$

where the capacity $c_{n}$ depends on the size of armatures and their gap, all considered equal. $v_{2}, v_{3}$ are opposite in sign and equal in magnitude, assigned to a control voltage $v_{n}$ such that $v_{3}=v_{n}, v_{2}=-v_{n}$, and $v_{1}$ is a fixed control.

Thus, the force provided by the actuator is proportional to $v_{n}$ and $v_{1}$ can be used to adjust the actuator constant. It is important to note that this force does not depend on the position of the probe on the normal direction, as long as the overlapping surface on mobile and fixed armatures is constant, as guaranteed by design. This is a considerable benefit over flexible systems with intrinsic dependencies between force and displacement.

\section{Mechanical behavior}

The dynamic of the system is well described by a second order differential equation involving all forces applied to the probe,

$$
f=m \ddot{x}-a_{n}+k x+b \dot{x}
$$

where $f$ is the interaction force between probe and sample, $m \ddot{x}$ is the inertial term, $a_{n}$ is the force provided by the actuator, $k x$ is the force due to suspension compliance, $b \dot{x}$ is the viscous damping term.

\section{Prototype}

The meso-scale prototype illustrated in Fig 5 is comprised a frame made of polyoxymethylene plastic (180 $\mathrm{mm} \times 180 \mathrm{~mm} \times 40 \mathrm{~mm}, 10 \mathrm{~mm}$ thickness) supporting the fiber positioning and tensioning mechanisms and the fixed actuator armatures. The carrier is machined out of magnesium to minimize mass $(1.2 \mathrm{~g})$. Its dimensions are $22 \mathrm{~mm} \times 8.5 \mathrm{~mm} \times 7.4 \mathrm{~mm}$ and a gap between fixed and moving armatures of $0.3 \mathrm{~mm}$. The springs are made out pinchbeck. Care was exercised to minimize hysteresis by using long glass fibres (180 $\mathrm{mm}$ longer, $0.1 \mathrm{~mm}$ diameter) and careful fiber attachment design. The probe is made from pulled glass tube of $1 \mathrm{~mm}$ in diameter (Sutter glass puller, PI1000). To measure the displacements of the carrier, a laser (Camero, $4 \mathrm{~mW}, 635 \mathrm{~nm}$ wavelength) and a quadrant photodiode detector (Silicon Sensor, QP5.8-6 SD) are placed behind the frame. 


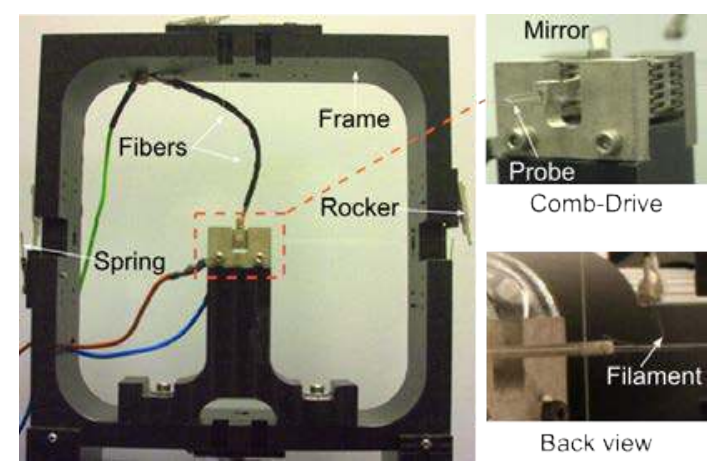

Fig. 5. Mechanical elements.

The electrical connection to the moving armature actuator is provided by a tungsten incandescent lamp filament (Fig. 5). The voltages applied to the fixed armatures, $v_{n}$ and $-v_{n}$, were provided by two high voltage operational amplifiers $( \pm 200 \mathrm{~V}$, APEX, PA82J). The reference for each amplifier are digitally set through a DAC/ADC card (National Instrument, PCI6259) and the control algorithm is implemented on a PC running RTAI real-time Linux kernel. The sampling rate is set to $1 \mathrm{kHz}$.

\section{E. Prototype calibration}

The calibration of the system as a force sensor requires three parameters: the optical lever calibration constant, the stiffness of the suspension, and the electromechanical constant of the actuator. Moreover, dynamic properties of the sensor are investigated by experimental identification.

1) Optical lever: Fig. 6 shows the position of the carrier as a function of the output voltage, $U_{h}$, of the photodiode and $U_{h}$ as a function of the excitation voltage, $U$. Experimental data were fitted with straight lines. Calling $k_{1}$ and $k_{2}$ the calibration constants of the photodiode and of the actuator respectively, led to $k_{1}=387 \mu \mathrm{m} / \mathrm{V}$ and $k_{2}=0.0274$. The relation between the $U_{h}$ and $U$ is linear as expected.

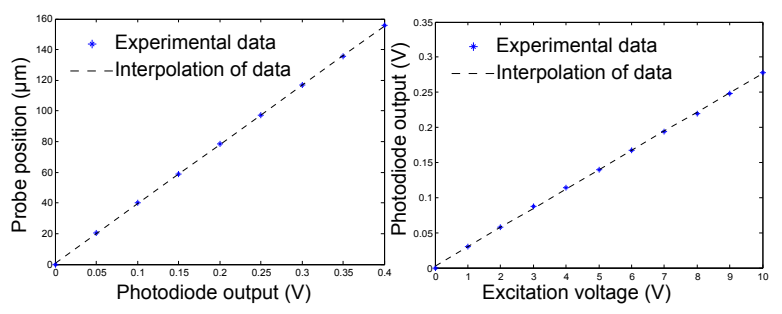

Fig. 6. Photodiode and actuator responses.

2) Stiffness: The suspension stiffness is found by measuring the natural frequency of the mass-spring system and the known mass of the carrier. For the particular tuning used in the experiments described in the Section $\mathrm{V}$, the measured natural frequency is $9.4 \mathrm{~Hz}$. Given that the natural frequency is given by $f_{0}=\frac{1}{2 \pi} \sqrt{\mathrm{k} / m}$ and the mass is $m=1.2 \mathrm{~g}$, the stiffness, $k$, is $3.83 \mathrm{~N} / \mathrm{m}$.

3) Actuator: At rest, the actuator force balances the elastic force of the suspension, thus $a_{n}=k k_{1} k_{2} U$. According to this expression the actuator constant is equal to $k k_{1} k_{2}$, here $k_{a}=40.68 \mu \mathrm{N} / \mathrm{V}$. The force produced by the actuator is indeed found to be proportional to the input voltage, validating its linear behavior.

\section{F. Identification}

As shown in Section II-A, in the absence of interaction force $f$, the mechanical behavior of the system is described by

$$
m \ddot{x}-a_{n}+k x+b \dot{x}=0
$$

that is a system of order two, with a natural pulsation, $w_{n}$, and damping coefficient, $\zeta$. The Laplace transfer function corresponding to this system is given by

$$
G(s)=\frac{k_{n} w_{n}^{2}}{s^{2}+2 \zeta w_{n} s+w_{n}^{2}},
$$

where $k_{n}, w_{n}$ and $\zeta$ are to be defined. The system is identified from a step response and the values $k_{n}=0.032, \zeta=0.0047$, for $w_{n}=64 \mathrm{rad} / \mathrm{s}$, are found (step reference is fixed to 4 volts and the identification is carried out with the Matlab system identification tool).

\section{ACTIVE CONTROL MEASUREMENT PRINCIPLE}

With passive sensing, the force is provided by measuring the deformation of a structural element. As the objective here is to detach the measurement from a structural stiffness, an active control approach is applied. It consists in opposing, by an artifice, the value of the force to be measured with an adjustable value of the same or different nature, in the objective of immobilizing the probe at its equilibrium position. In this case, the force measurement is not computed using the signal coming from the displacement sensor, but the value of the opposed artifice used to balance the force.

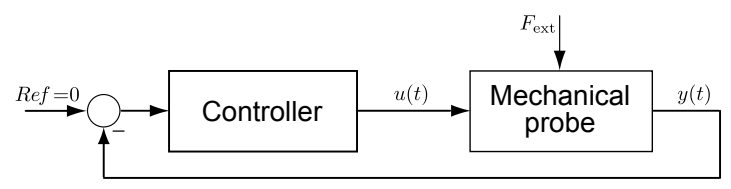

Fig. 7. Active force sensing principle. $F_{\text {ext }}, y(t)$ and $u(t)$ correspond respectively to $f, x$ and $U$ defined in section II.

Figure 7 illustrates the general principle of this active force measurements method. The interaction force $F_{\text {ext }}$ appears as a perturbation to be rejected by the controller in order to maintain the sensitive part of the sensor at its equilibrium position. This compensation provides directly the measure of $F_{\text {ext }}$ which is proportional to the control signal $u(t)$. This principle has many advantages. It improves the precision, increases the force measurement range and improves both, robustness and stability of the sensor.

\section{IV. $\mathrm{H}_{\infty}$ OPTIMAL CONTROL}

This section deals with the design of the H-infinity optimal control. As a systematic method, $\mathrm{H}_{\infty}$ synthesis provides a framework for the control of linear system from the $\mathrm{H}_{\infty}$ norm and frequency concepts. It allows to design dynamic controller using the available measure by taking into account the robust stability and the performances specifications in the same time. 


\section{A. $H_{\infty}$ standard problem}

$\mathrm{H}_{\infty}$ synthesis uses the standard problem formulation [18], [19] shown in Fig. 8. Where $P(s)$ is the generalized transfer matrix modelling the dynamic interaction between inputs/outputs, including the weighting functions. $K(s)$ is the controller to design. $w$ is the exogenous input containing disturbance, noise and reference, $u$ is the control signal, $e$ is the output to supervise usually comprising the error signal and weithed control input, $z$ is the measure signal available for feedback.

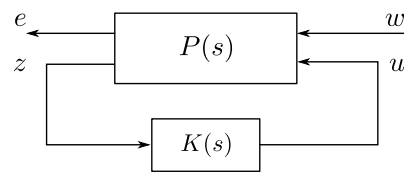

Fig. 8. Standard problem.

The standard $\mathrm{H}_{\infty}$ optimisation problem consists to find a proper controller $K(s)$ in order to stabilize the closedloop internally and to minimize the $\mathrm{H}_{\infty}$ norm of the transfer function from $w$ to $e$. More details are given in [18], [20], [21] about $H_{\infty}$ problem and method used to resolve it.

\section{B. $H_{\infty}$ synthesis}

In order to use $\mathrm{H}_{\infty}$ technique, the original problem (see Fig. 7) is converted to the standard problem given in Fig. 8 by performing some block diagram manipulations as shown in Fig. 9. Since the reference is set to zero and the perturbation acts on the output in this case, two weighting functions $W_{1}$ and $W_{2}$ are used to get adjustable parameters for the control design. Compared to Fig. 8, $w$ consists of external perturbation $d, z$ consists of the measured signal $y, e$ consists of the supervised signals $e_{1}$ and $y, u$ is the control signal. The synthesis requires to find rational optimal function $K(s)$ to stabilize the closed-loop, by minimizing the transfer function from $d$, (external force perturbation) to $e_{1}, e_{2}$ (signals to supervise) given by

$$
\left\|\begin{array}{c}
W_{1}(s) S(s) \\
-W_{1}(s) W_{2}(s) K(s) S(s)
\end{array}\right\|_{\infty}<\gamma
$$

where $S$ is the sensitivity function $\left(S(s)=(I+G(s) K(s))^{-1}\right)$. The weighing functions, $W_{1}(s)$ and $W_{2}(s)$, respectively ap-

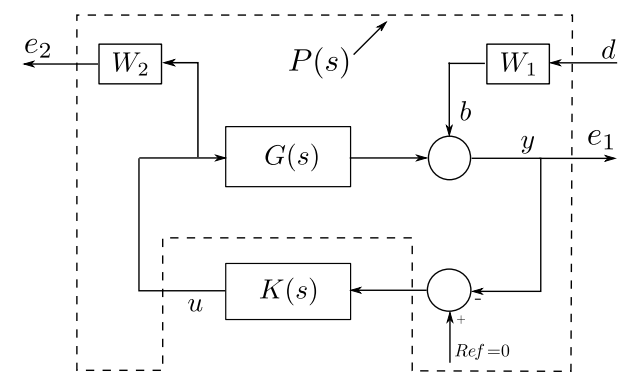

Fig. 9. Set up to determine a controller from $H_{\infty}$ optimal minimization.

plied to the input signal $d$ and the control signal $u(t)$, are selected to specify the performance that the controller should achieve [18]. The weighing function $W_{1}$ specifies an error of about $0.1 \%$ in the low frequencies, a modulus margin of 0.5 , and a bandwidth of $60 \mathrm{rad} / \mathrm{s} . W_{2}$ specifies the control signal constraints. In fact, control inputs must be restricted because of the limited control energy and actuator saturation.

$$
W_{1}(s)=\frac{0.5 s+60}{s+0.006}, \quad W_{2}(s)=0.01
$$

The $H_{\infty}$ controller, $K(s)$ stabilizing the closed-loop is then found by minimizing the bound of the $H_{\infty}$-norm given in (5). The $\mathrm{H}_{\infty}$ problem is resolved using the LMI implemented in Matlab Robust control Toolbox [22]. A controller of order 3 was obtained with $\gamma$ of $0.9647(<1)$. The resulting open-loop system response is shown in Fig. 10, providing interesting gain and phase margins, $63.5 \mathrm{~dB}$ and $70.2 \mathrm{deg}$ respectively.

$$
K(s)=\frac{7.92810^{6} s^{2}+4.30610^{6} s+2.79610^{10}}{s^{3}+9.79610^{4} s^{2}+1.67510^{7} s+9.9710^{4}}
$$

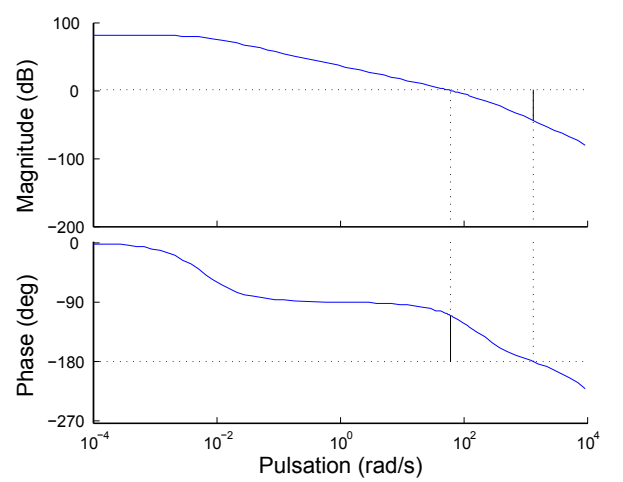

Fig. 10. Bode diagram of the open loop of the system.

The discrete time transfer function $K(z)$ using a Zero-order hold method with sampling period $h=1 \mathrm{~ms}$ is given below. This function is computable in real-time with reasonable computational effort. This controller is implemented on a PC running Linux operating system patched with the RTAI ${ }^{1}$ (Real Time Application Interface) to provide real time functionality.

$$
K(z)=\frac{73.26 z^{3}-72.96 z^{2}-73 z+73.22}{z^{3}-0.8823 z^{2}-0.9262 z+0.8084}
$$

\section{EXPERIMENTAL VALIDATION OF THE ACTIVE SENSOR}

Experimental validation aims to verify the performances of the active measurement principle described above. As the bandwidth of the present sensor remains low compared to the dynamic of van der Waals forces, the validation is carried out by measuring attractive magnetic forces. This study case is driven by two reasons: The magnetic force increases rapidly according to the distance as van der Waals forces by distance ${ }^{-2}$ or $^{-3}$. Magnetic forces are of a higher amplitude of van der Waals, less sensitive to environment parameters and controlled much more easily. By using a permanent magnet, the force dynamic can be adapted to

\footnotetext{
${ }^{1}$ https://www.rtai.org/
} 
the sensor bandwidth, providing an ideal case to validate its principle.

\section{A. Experimental set-up}

The magnetic force exerted on an iron particle by a permanent magnet is studied. A $1 \mathrm{~mm} \times 1.5 \mathrm{~mm} \times 5 \mathrm{~mm}$ permanent magnet and a $2.06 \mathrm{~mm}$ of diameter, $1.27 \mathrm{~mm}$ thickness iron particle are used. The magnet is mounted on a needle, which can be displaced by a micro-stage (see Fig. 12). The particle is attached to the sensor probe as shown in Fig. 11.

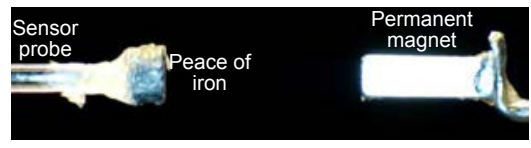

Fig. 11. Experimental setup to measure the interaction between a permanent magnet and an iron particle

To measure the magnetic force acting on the particle, the experimental bench depicted in Fig. 12 is mounted. It is comprised of a motorized $x y z$ micro-positioning stage for coarse motion (Sutter MP-580) and an electromagnetic transducer for fine motion for the sample motion, measured by an interferometer (SIOS SP-120). A microscope (VehoVMS-004) is used for visual feedback. The active probe and its laser optical lever are fixed. The bench is placed on an anti-vibration table to avoid external perturbations.

To limit sensor sensitivity to environmental conditions, the experimental bench is placed in a clean room with controlled temperature and humidity. Furthermore, the bench is placed inside a box to avoid acoustic and air conditioning perturbations.

\section{B. Results}

The magnetic force is measured on two passes, 'approach' (toward the magnet) and 'retract'. The permanent magnet is initially placed at distance of $6 \mathrm{~mm}$ from the iron particle. At this position, the magnetic force exerted by the magnet is negligible. From this initial position, the magnet is approached with a constant velocity of $0.4 \mathrm{~mm} / \mathrm{s}$ until the sensors reaches its absolute upper force limit around $\sim 400 \mu N$, at a distance of $0.6 \mathrm{~mm}$, then retracted with the same velocity. The force on the particle due to the field of the permanent magnet is measured by the sensor by balancing this force in real time. Figure 13 represents the measured magnetic force in the horizontal direction versus relative distance. The curve shows that, as the distance decrease, the force increase rapidly as distance ${ }^{-2}$. It is also clear from the curve that the sensor captures the whole magnetic force behavior and dynamic during the approach and retract with no instabilities or hysteresis: the measurement at approach and retract cycles are virtually identical. This preliminary result shows the effectiveness and the efficiency of the developed active probe and validates the potential of the active principle measure.

Furthermore, to estimate the resolution of the sensor a virtual perturbation is applied on the sensing position of the

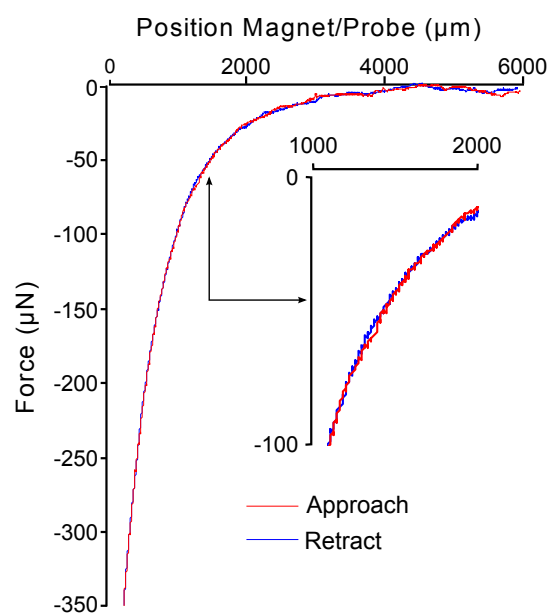

Fig. 13. Magnetic force vs position.

probe. According to the variation of this perturbation, the significant variation of the force sensed by the prototype starts from $0.4 \mu \mathrm{N}$. That means that the smallest force which can be measured by the sensor is $0.4 \mu \mathrm{N}$. This value corresponds then to the resolution achieved by the present sensor.

\section{Discussion}

An active force sensor was proposed. It is designed around a glass fiber suspension, a differential bipolar comb actuator and an active force measurement principle. It is distinct from previous designs in several aspects. Actively controlled electrostatic forces are used instead of bending stresses or structural stiffness to balance the force acting on the sample. The suspension is arranged to constrain movements accurately to a single degree of freedom in translation. An electrostatic actuator combined with an optical lever for position feedback through an especially designed controller yields a large range of measurements both in force and displacement. Based on the feedback through $\mathrm{H}_{\infty}$ controller, the actuator generates an opposite balance force to cancel the action of the interaction forces. The measurement is related to the balanced force which makes the probe remains at equilibrium position. Preliminary experimental results verify the performances of the active force sensor and its effectiveness. Table I resumes the main proprieties of the designed force sensor.

TABLE I

FORCE SENSOR PROPERTIES

\begin{tabular}{ll}
\hline Property & Remark \\
\hline Stability & good margin \\
\hline Resolution & estimated to $0.4 \mu \mathrm{N}$ \\
\hline Measurement range & $-400 \mu \mathrm{N}$ to $400 \mu \mathrm{N}$ \\
\hline Bandwidth & $60 \mathrm{rad} / \mathrm{s}$ \\
\hline
\end{tabular}

The sensor is validated by measuring the variation of magnetic forces over $10 \mathrm{~mm}$ range up to $350 \mu \mathrm{N}$. The measurement reproduces perfectly the magnetic force profile 


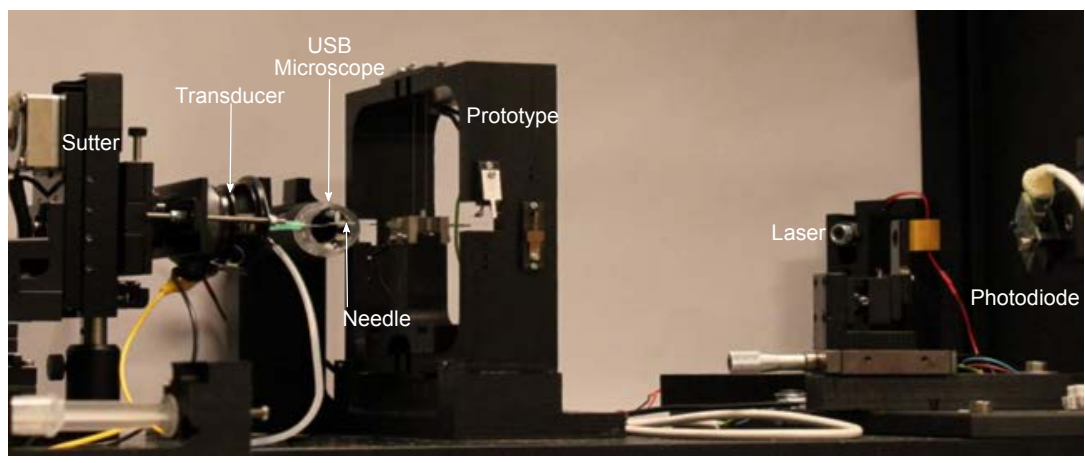

Fig. 12. Experimental bench.

as a function of distance ${ }^{-2}$, without hysteresis and regardless of displacement direction.

\section{CONCLUSIONS}

In this paper a H-infinity optimal controller is used for micro-force sensing. A micro-force sensor was designed around a linear comb-drive actuator with a balance force behavior. Based on the feedback of the position of the probe, the H-infinity controller generates an opposite force to balance the external applied force to maintain the probe at its equilibrium position. As the result, the force measurement is not computed using the signal coming from the displacement of the probe, but proportional to the value of the opposite voltage applied by the controller. Furthermore, the closedloop control allows to maintain the sensitive part of the sensor in linear range, improve precision, increase the sensor dynamic range and improve robustness and stability of the sensor.

Future work would improve fabrication to increase the sensor bandwidth, while retaining the same principle. To this end, MEMS fabrication techniques could be applied advantageously with drastic reduction in size and mass. The bandwidth of the micro-scale active sensor is then expected to reach the $\mathrm{kHz}$ range.

\section{ACKNOWLEDGMENTS}

The authors wish to thank Sheng Chao WONG for advise and help on electronic design. This work has been supported by the French National Project ROBOTEX ANR-10-EQPX44-01 and Emergence-UPMC 2011 research program.

\section{REFERENCES}

[1] M. Gauthier and S. Régnier. Robotic Micro-assembly, IEEE press, publisher, 2010.

[2] S. Régnier and N. Chaillet. Microrobotics for Micromanipulation, Wiley-ISTE, publisher, 2010.

[3] S. Alvo, P. Lambert, M. Gauthier and S. Régnier. A van der Waals Force-Based Adhesion Model for Micromanipulation, Journal of Adhesion Science and Technology, Special Issue on Adhesion in MEMS/NEMS, Vol. 24, No 15-1, pp. 2415-2428, 2010.

[4] A. Bolopion, B. Cagneau, S. Haliyo and S. Régnier. Analysis of stability and transparency for nanoscale force feedback in bilateral coupling, Journal of Micro-Nano Mechatronics, Vol 4, pp. 145-158, 2009.

[5] G. Lin, R.E. Palmer, K.S. Pister and K.P. Roos. Miniature Heart Cell Force Transducer System Implemented in MEMS Technology, IEEE Transactions on Biomedical Engineering. Vol. 48, No 9, pp. 996-1006, September 2001
[6] M. Sepaniak, P. Datskos, N. Lavrik and C. Tipple. Microcantilever transducers: A new approach in sensor technology. Analytical chemistry, pp. 568-575, 2002.

[7] F. Arai, A. Kawaji, T. Sugiyama, Y. Onomura, M. Ogawa, T. Fukuda, $\mathrm{H}$. Iwata and $\mathrm{K}$. Itoigawa. $3 \mathrm{~d}$ micromanipulation system under microscope, In International Symposium on Micromechatronics and Human science. pp. 127-134, 1998.

[8] Y. Sun, W. Kai-Tak, K. Roberts, J. Bischof and J. Bradley. Mechanical property characterisation of mouse zona pellucida, IEEE Transactions on nanobioscience, Vol. 2, $\mathrm{N}^{\circ} 4$, pp. 279-285, 2003

[9] W. Li and N. Xi. Novel micro gripping, probing and sensing devices for single-cell surgery, In Proceeding of the 26th international conference of the IEEE EMBS, pp. 2591-2594, 2004.

[10] P. Rougeot, S. Régnier and N. Chaillet. Forces analysis for micromanipulation, In Proceeding 2005 IEEE international symposium on computational intelligence in robotics and automation, pp. 105-110, 2005.

[11] N. Kato, I. Suzuki, H. Kikuta and K. Iwata. Force-balancing microforce sensor with an optical-fiber interferometer. Review of scientific instruments, Vol.68, pp. 2474-2478, 1997.

[12] F. Beyeler, A. Neild, S. Oberti, D. J. Bell, Y. Sun, J. Dual and B. J. Nelson. Monolithically Fabricated Microgripper With Integrated Forces Sensor for Manipulating Microobjects and Biological Cells Aligned in an Ultrasonic field, Journal of Microelectromechanical Systems, Vol. 16, No 1 , pp. 07-15, February 2007.

[13] K. H. Chau, S. R. Lewis, S. F. Bart, R. G. Marcheselli, and R. T. Howe. An integrated force-balanced capacitive accelerometer for lowG applications, Sens. Actuators A Phys., Vol. 54, pp, 472-276, 1996.

[14] B. P. Gogoi and C. H. Mastrangelo. A Low-voltage Force Balanced Pressure Sensor with Hermetically Sealed Servomechanism, Micro Electro Mechanical Systems, MEMS'99, pp. 493-498, 1999.

[15] Y. Sun, D. P. Potasek, D. Piyabongkarn, R. rajamani and B. J. Nelson. Actively servoed multi-axis microforce sensors, in Proc; ICRA, pp. 294-299, 2003.

[16] Y. Shen, E. Winder, X. Ning, C. A. Pomeroy and U. C. Wejinya Closed-Loop Optimal control-Enabled Piezoelectric Microforce Sensors, IEEE/ASME TRANSACTIONS ON MECHATRONICS, Vol. 11, $\mathrm{N}^{\mathrm{o}} 4$, pp. 420-427, August 2006.

[17] D. Sidobre and V. Hayward. Calibrated measurement of the behaviour of mechanical junctions from micrometre to subnanometre scale : the friction force scanner, Meas. Sci. Technol. Vol. 15, pp, 451-459, 2004.

[18] X. P. Li, and B. C. Chang. An $H_{\infty}$ design for aircraft longitudinal flight control, First IEEE Conference on Control Applications, Vol. 2, pp. $596-601,1992$.

[19] Y. Wei-qian; C. Huai-hai and H. Xu-dong. Tracking control research of high-order flexible structures on the H-infinity control method, 2nd International Conference on Advanced Computer Control (ICACC), Vol.5, pp. 111-115, 27-29 March 2010.

[20] X. P. Li, B. C. Chang, S. S. Banda and H. H. Yeh. Robust control system design using $\mathrm{H}_{\infty}$ optimisation theory, Journal of guidance control and dynamic, Vol. 15, Nº4, pp. 944-952, 1992.

[21] K. Glover. All optimal hankel-norm approximations of linear multivariable systems and their $l^{\infty}$ error bounds. Int. Journal of Control, Vol. 39, pp. 1115-1193, 1984.

[22] P. Gahinet and P. Apkarian. A linear matrix inequality approach to $\mathrm{H}_{\infty}$ control, International Journal of Robust and Nonlinear Control, vol. 4, pp. 421-448, 1994. 